\title{
Problematik der Kostenerstattung für Akupunkturbehandlungen auf Kassenschein
}

\author{
Klaus Trinczek \\ Allgemeinarzt, Akupunktur - Chirotherapie - Naturheilverfahren, Erlangen, Deutschland
}

\section{Schlüsselwörter \\ Akupunktur $\cdot$ Kostenerstattung $\cdot$ Kassenschein}

\begin{abstract}
Zusammenfassung
Akupunktur ist in Deutschland seit 2007 eine Leistung der gesetzlichen Krankenversicherung. Allerding ist diese auf 2 Diagnosen eingegrenzt ("chronische Schmerzen der Lendenwirbelsäule» und "chronische Schmerzen der Kniegelenke durch Gonarthrose») und mit semistandardisierten Vorgaben zur Durchführung von Akupunkturbehandlungen verbunden, die teilweise nicht den Weiterbildungsinhalten der Akupunkturausbildung entsprechen. Akupunktur wird vor allem in orthopädischen Praxen angewandt. Hohe Anforderungen an die Qualifizierung und Qualitätssicherung sowie die Schwierigkeit, die Behandlungen wirtschaftlich durchführen zu können, schmälern die Attraktivität, Akupunktur in der gesetzlichen Krankenversicherung zu verankern.
\end{abstract}

\section{Einführung}

Wofür sich ärztliche Akupunkteure in den letzten Jahrzehnten engagiert haben, ist heute Realität: Akupunktur ist eine Behandlungsmethode, die durch eine offizielle Zusatzbezeichnung der Ärztekammern und als Leistung der gesetzlichen Krankenversicherung (GKV) anerkannt ist. Aber hat die Akupunktur ihren Weg von einer Außenseitermedizin in die Mitte der kassenärztlichen Behandlungswirklichkeit tatsächlich gefunden?

Um den Stellenwert der Akupunktur in der GKV einschätzen zu können, ist ein Blick zurück zu den Anfängen hilfreich. Akupunktur war in den 1990er-Jahren immer stärker in den Fokus der

\author{
Keywords \\ Acupuncture - Reimbursement . \\ Statutory health insurance
}

\section{Summary \\ Reimbursement for Acupuncture Treatments in the German Statutory Health Insurance System}

Since 2007, acupuncture in Germany is reimbursed by the statutory health insurance. However, this policy is limited to the diagnoses 'chronic pain in lumbar spine' and 'chronic pain in knee joint through gonarthrosis' and connected to semi-standardized specifications for acupuncture treatment that partly do not correspond with standards in professional acupuncture training. Predominantly, acupuncture is applied in orthopedic practice. High demands in qualification and quality assurance as well as the difficulty to establish an economically attractive framework in acupuncture treatment are issues that curtail the appeal of acupuncture as treatment option to be offered by statutory health insurance.

Öffentlichkeit gerückt. Immer häufiger wurden auch Versicherte der GKV auf privatärztlicher Grundlage akupunktiert. Die Behandlung wurde auf Antrag von den Kassen erstattet.

Der AOK-Bundesverband strebte nicht zuletzt wegen der zunehmenden Anzahl von Anträgen auf Kostenerstattung eine grundsätzliche Klärung an, ob die Kosten für Akupunkturbehandlungen von den gesetzlichen Krankenkassen übernommen werden dürfen. Er stellte im Mai 1998 beim obersten Entscheidungsgremium der Selbstverwaltung in der GKV, dem Gemeinsamen Bundesausschuss der Ärzte und Krankenkassen (G-BA) den Antrag, Akupunktur solle als Behandlungsmethode hinsichtlich Indikation, Nutzen, medizinischer Notwendigkeit und Wirtschaftlichkeit beurteilt werden.

\begin{tabular}{ll}
\hline KARGER & ( ) 2015 S. Karger GmbH, Freiburg \\
& $1661-4119 / 15 / 0222-0118 \$ 39.50 / 0$ \\
Fax +49 761 452 07 14 & Accessible online at: \\
Information@Karger.com & www.karger.com/fok \\
www.karger.com &
\end{tabular}


Im Jahr 2001 veröffentlichte der eingesetzte Arbeitsausschuss die Ergebnisse der Beratungen in einem 475 Seiten umfassenden Bericht [1]. Der Ausschuss sah die Qualität der bisherigen Studien in der Akupunkturforschung als methodisch mangelhaft an. Zudem gäbe es Hinweise auf eine Veröffentlichung von tendenziösen Studienergebnissen (publication bias). Es würden nicht alle Studien veröffentlicht, die eine mangelnde Wirksamkeit von Akupunktur zeigen. Interessant ist, dass diese Kritik auch den Ergebnissen der amerikanischen Konsensus-Konferenz des National Institute of Health (NIH) gilt. Diese waren 1998 im Journal of the American Medical Association (JAMA) veröffentlicht worden und werden auch heute häufig als Meilenstein auf dem Weg der wissenschaftlichen Anerkennung der Akupunktur bewertet [2]. «Der Arbeitsausschuss stellt zusammenfassend fest, dass die Schlussfolgerungen der NIH-Konsensus-Konferenz vom November 1997 weit über den wissenschaftlichen Kenntnisstand hinausgehen und nicht durch adäquate Studien belegt sind» [1].

Der G-BA empfahl wegen der aus seiner Sicht nicht aussagekräftigen Studienlage im Oktober 2000 die Durchführung von Modellverfahren zur Erforschung der Akupunkturwirkung für folgende Indikationen: chronische Kopfschmerzen, chronische Schmerzen der Lendenwirbelsäule (LWS) und chronische Schmerzen bei Osteoarthritis.

Es wurden 2 Reihen von Modellprojekten mit dreiarmigen Studien initiiert: die GERAC-Studien (German Acupuncture Trials) unter der Leitung des AOK-Bundesverbandes (wissenschaftliche Leitung Prof. Trampisch, Ruhr-Universität Bochum) und die ART/ARC-Studien (Acupuncture Randomised Trials/Acupuncture in Routine Care Studies) der Ersatzkrankenkassen (Wissenschaftliche Leitung Prof. Melchart, Technische Universität München, ART) in Kooperation mit der Charité Berlin (wissenschaftliche Leitung Prof. Willich, ARC), finanziert von der Techniker Krankenkasse und der dem Modellprojekt beigetretenen Kassen.

\section{Der Beschluss des Gemeinsamen Bundesausschusses}

In den Modellprojekten konnte die Wirksamkeit von Akupunktur nachgewiesen werden. Dennoch wurden die Ergebnisse kontrovers diskutiert. Das lag insbesondere an den fehlenden Wirksamkeitsunterschieden zwischen Verum-Akupunktur (Verwendung von Punkten auf Grundlage der chinesischen Medizin) und ShamAkupunktur (oberflächliche Nadelung ohne Stimulation an Punkten außerhalb des Leitbahnsystems). Bei der Anerkennung der Akupunktur als Kassenleistung war für den G-BA jedoch entscheidend, dass die Überlegenheit der Behandlung mit Akupunkturnadeln (Verum- und Sham-Akupunktur) gegenüber der Standardtherapie evident war. Der G-BA beschloss daher 2006, Akupunktur bei den Diagnosen «chronische Schmerzen der LWS» und «chronische Schmerzen in mindestens einem Kniegelenk durch Gonarthrose» als Behandlungsmethode anzuerkennen [3]. Damit war Akupunktur ab 01.01.2007 Leistung der GKV.

\section{Die Definition von Akupunktur als Kassenleistung}

Insgesamt bleibt es bei der grundsätzlichen Ablehnung der Akupunktur als vertragsärztliche Leistung der GKV. Akupunktur wird nur unter bestimmten Voraussetzungen als Behandlungsform anerkannt, d.h. ausschließlich «Körperakupunktur mit Nadeln ohne elektrische Stimulation bei chronisch schmerzkranken Patienten», wobei differenzierte Vorgaben zu Indikation, Krankheitsbild, Durchführung der Akupunktur, Qualifikation und Qualitätssicherung zwingend vorgegeben sind. Somit ergeben sich die folgenden Voraussetzungen.

\section{Zugelassene Indikationen}

1. Chronische Schmerzen der LWS, die seit mindestens 6 Monaten bestehen und gegebenenfalls nicht-segmental bis maximal zum Kniegelenk ausstrahlen (pseudoradikulärer Schmerz) - mit jeweils bis zu 10 Sitzungen innerhalb von maximal 6 Wochen und in begründeten Ausnahmefällen bis zu 15 Sitzungen innerhalb von maximal 12 Wochen, jeweils mindestens 30 min Dauer, mit jeweils 14-20 Nadeln.

2. Chronische Schmerzen in mindestens einem Kniegelenk durch Gonarthrose, die seit mindestens 6 Monaten bestehen - mit jeweils bis zu 10 Sitzungen innerhalb von maximal 6 Wochen und in begründeten Ausnahmefällen bis zu 15 Sitzungen innerhalb von maximal 12 Wochen, jeweils mindestens 30 min Dauer, mit jeweils 7-15 Nadeln pro behandeltes Knie.

\section{Qualifikationsvoraussetzungen}

Für Vertragsärzte sind Qualifikationsvoraussetzungen formuliert:

1. Nachweis der Zusatzbezeichnung Akupunktur der Bundesärztekammer;

2. Nachweis von Kenntnissen in der Psychosomatischen Grundversorgung (80-h-Curriculum);

3. Nachweis der Teilnahme an einem interdisziplinärem Kurs zur Schmerztherapie $(80 \mathrm{~h})$.

\section{Qualitätssicherung}

Als weitere Anforderungen werden Merkmale der Qualitätssicherung angegeben:

1. Einbindung der Akupunktur in ein schmerztherapeutisches Gesamtkonzept mit Erstellung eines Therapieplans;

2. Eingangs- und Verlaufserhebung zur Schmerzevaluation mit schmerztherapeutischen Parametern;

3. Stichprobenartige Überprüfung dieser Erhebungen durch eine Kommission der Kassenärztlichen Vereinigung (KV);

4. Teilnahme an Fallkonferenzen bzw. Qualitätszirkeln;

5. Voraussetzungen für Räumlichkeiten (Akupunktur muss in separaten, abgeschlossenen und mit einer Liege ausgestatteten Räumen durchgeführt werden);

6. Es dürfen nur sterile Einmalnadeln verwendet werden [4].

Nur unter diesen Voraussetzungen und bei Erfüllung aller Vorgaben bis hin zur Anzahl der verwendeten Nadeln gilt Akupunktur als Kassenleistung. 
Tab. 1. Akupunktur gemäß Qualifikationsgebundenen Zusatzvolumina (QZV) der Kassenärztlichen Vereinigung Hamburg (KVH) für das 3.Quartal 2013 [6]

\begin{tabular}{lcc}
\hline Fachgruppe & QZV-Fallwert, EUR & Durchschnittliche QZV-Fallzahl, n \\
\hline Allgemeinmedizin & 138,21 & 21 \\
Orthopädie & 83,64 & 166 \\
Neurologie & 150,06 & 64 \\
Physikalische und Rehabilitative & 158,80 & 103 \\
$\quad$ Medizin & & \\
Schmerztherapeuten & 124,84 & 27 \\
\hline
\end{tabular}

\section{Die Vergütung von Akupunkturleistungen}

Die wirtschaftliche Bewertung unterliegt verschiedenen Einflussgrößen. Grundsätzlich ist die Höhe der Vergütung im Einheitlichen Bewertungsmaßstab (EBM) der Kassenärztlichen Bundesvereinigung (KBV) festgelegt. Über die Qualifikationsgebundenen Zusatzvolumina (QZV) erfolgt aber eine sogenannte Mengensteuerung. In der Hausarztzentrierten Versorgung $(\mathrm{HzV})$ gelten wiederum eigene Bedingungen.

\section{Einheitlicher Bewertungsmaßstab}

Betrug die Vergütung im Rahmen der Modellprojekte noch 35 EUR pro Akupunkturbehandlung, findet sie sich heute im EBM als Abrechnungsziffer 30791 mit 21,78 EUR wieder. Das entspricht einem Minus von 37\%. Zusätzlich kann die Ziffer 30790 für die Eingangsdiagnostik und Abschlussuntersuchung maximal 1-mal pro Jahr abgerechnet werden. Sie wird mit 48,28 EUR vergütet.

Im Gegenzug verliert der akupunktierende Kassenarzt allerdings auch Honorar an anderer Stelle. Akupunktur ist im EBM dem «Abschnitt 30.7 Schmerztherapie» zugeordnet und gilt als «Spezialleistung». Damit können die «Grundversorgungspauschalen» zur Förderung der Basisversorgung in den einzelnen Fachgebieten nicht mehr abgerechnet werden. Im hausärztlichen Bereich wird weder die Hausärztliche Vorhaltepauschale und der seit 01.01.2015 geltende Zuschlag (EBM-Ziffern 03040 und 03060) mit einem Wert von zusammen 17,06 EUR pro Quartal vergütet, noch können die Chroniker-Pauschalen (EBM-Ziffern 03220 und 03221) mit maximal 17,46 EUR pro Quartal abgerechnet werden. Im fachärztlichen Bereich kann die Pauschale zur Förderung der fachärztlichen Grundversorgung und deren Zuschlag nicht geltend gemacht werden. Für die Fachgebiete Anästhesie, Chirurgie, Orthopädie und Physikalische Medizin liegt der Wert dieser Pauschalen zwischen 4,21 und 8,43 EUR pro Quartal [5].

Erbringt also ein Arzt auch nur eine Akupunkturbehandlung, dann verliert er im gleichen Quartal an anderer Stelle Einnahmen: Hausärzte bis zu 34,52 EUR pro Quartal und die Fachärzte, die üblicherweise Akupunktur zulasten der GKV durchführen, bis zu 8,43 EUR pro Quartal.

\section{Qualifikationsgebundene Zusatzvolumina}

Was die Honorierung angeht, ist wesentlich, dass nicht jede Akupunktur in vollem Umfang bezahlt wird. Akupunkturleistungen sind für jede Praxis begrenzt. Sie unterliegen einer Mengen- steuerung durch die QZV. Die Berechnung der QZV wird in den KV der einzelnen Bundesländer unterschiedlich gehandhabt. Gemeinsam ist, dass sie für jede Arztgruppe getrennt berechnet werden. Die Höhe ist abhängig von der für das QZV zur Verfügung stehenden Geldmenge und der Anzahl der erbrachten Leistungen. Für jedes QZV gilt: Je mehr Leistungen innerhalb eines QZV abgerechnet werden, umso niedriger fällt der QZV-Fallwert aus, und die Vergütung der Einzelleistung bei gleichbleibender Behandlungsdichte sinkt. Bei einer Mengenausweitung von Akupunkturleistungen in einer Arztgruppe kommt es also zu einem Verfall der Vergütung. Auf diese Weise ergeben sich zum Teil erhebliche Verwerfungen zwischen den einzelnen Arztgruppen. Die KV Hamburg gibt im 3. Quartal 2013 für Fachärzte für Physikalische Medizin den QZV-Wert für Akupunktur mit 158,80 EUR an, während er für Orthopäden nur bei 83,64 EUR liegt (Tab. 1) [6].

\section{Hausarztzentrierte Versorgung}

Im Bundesland Bayern wird Akupunktur in fast allen vom Bayerischen Hausärzteverband selbst verhandelten Verträgen zur HzV als Einzelleistung in gleicher Höhe wie im KV-System vergütet. Das trifft aber nicht für alle Vereinbarungen der verschiedenen Bundesländer zu. In einigen Verträgen werden Akupunkturleistungen nicht gesondert vergütet. Sie sind Bestandteil einer Pauschale und gelten mit dieser als abgegolten. Beispiel ist der bundesweit geltende $\mathrm{HzV}$-Vertrag mit der Techniker Krankenkasse. Die «Kontaktabhängige Pauschale P2» wird über ein Jahr verteilt mit durchschnittlich 36,25 EUR pro Quartal vergütet [7]. Im sogenannten Ziffernkranz werden die Ziffern 30790 und 30791 als mit der Pauschale vergütet angegeben [8]. Dies führt zu der Situation, dass Akupunkturserien mit bis zu 15 Behandlungen mit durchschnittlich insgesamt nur 36,25 EUR bezahlt werden.

Die Vergütung von Akupunktur im Umfeld anderer Leistungen

Die Frage ist nun: Ist Akupunktur unterbezahlt? Ist sie innerhalb des EBM-Gefüges unterbewertet? Für diese Beurteilung ist der Vergleich mit Leistungen der Basisversorgung und apparativen Diagnostik hilfreich.

Für die gesamte hausärztliche Basisversorgung eines Quartals werden mit den hausärztlichen Versicherungspauschalen (EBMZiffern 03000 und 03040) altersabhängig zwischen 27,32 und 39,03 EUR vergütet. Orthopäden werden für die Basisversorgung ihres Fachbereichs (EBM-Ziffern 18210-18212 und 18220) altersabhängig mit Summen zwischen 21,16 und 24,75 EUR ausgestattet. 
Tab. 2. Honorarverteilung von Akupunkturleistungen auf Fachgruppen unter Verwendung von Daten der Kassenärztlichen Bundesvereinigung [9]

\begin{tabular}{|c|c|c|c|c|}
\hline $\begin{array}{l}\text { Verteilung von } \\
\text { Akupunkturleistungen }\end{array}$ & $\begin{array}{l}\text { Allgemeinmedizin/ } \\
\text { hausärztliche Internisten }\end{array}$ & Anästhesie & Orthopädie & Neurologie \\
\hline $\begin{array}{l}\text { Leistungshäufigkeit je Fall Ziffer } \\
30790 \text { (Diagnostik und Verlauf), \% }\end{array}$ & $<0,1$ & $<0,2$ & 2,4 & $<0,1$ \\
\hline $\begin{array}{l}\text { Leistungshäufigkeit je Fall Ziffer } \\
30791 \text { (Akupunkturbehandlung), \% }\end{array}$ & 1,8 & 6,0 & 22,7 & 1,8 \\
\hline $\begin{array}{l}\text { (a) Gesamtzahl der Ärzte, die eine } \\
\text { Grundpauschale abrechnen, n }\end{array}$ & 47204 & 1929 & 4314 & 530 \\
\hline $\begin{array}{l}\text { (b) Anzahl Akupunkturleistungen } \\
\text { abrechnender Ärzte, } \\
\text { n (\% der Gesamtzahl (a)) }\end{array}$ & $5.372(11,38)$ & $178(9,22)$ & $1,981(45,92)$ & $21(3,96)$ \\
\hline $\begin{array}{l}\text { (c) Durchschnittlicher Umsatz der } \\
\text { Fachgruppe in 2/2012, EUR }\end{array}$ & 47666 & 37758 & 53744 & 39570 \\
\hline $\begin{array}{l}\text { (d) Umsatzanteil der Akupunktur- } \\
\text { leistungen am Gesamthonorar } \\
\text { (c), \% / EUR }\end{array}$ & $0,5 / 238$ & $0,7 / 264$ & $11,0 / 5912$ & $0,6 / 237$ \\
\hline $\begin{array}{l}\text { (e) Gesamtumsatz der Fachgruppe } \\
\text { durch Akupunkturleistungen, } \\
\text { EUR }(\mathrm{a} \times \mathrm{d})\end{array}$ & 11234552 & 509256 & 25504368 & 125610 \\
\hline $\begin{array}{l}\text { (f) Umsatz durch Akupunktur- } \\
\text { leistungen pro akupunktierenden } \\
\text { Arzt, EUR (e/b) }\end{array}$ & 2091 & 2861 & 12874 & 5981 \\
\hline $\begin{array}{l}\text { Umsatzanteil durch Akupunktur- } \\
\text { leistungen pro akupunktierenden } \\
\text { Arzt (f), \% von (c) }\end{array}$ & 4,39 & 7,58 & 23,95 & 15,11 \\
\hline
\end{tabular}

Ein geplanter Regel-Hausbesuch (EBM-Ziffer 01410) wird mit 21,78 EUR gleich hoch bewertet wie eine Akupunkturbehandlung. Ein Belastungs-EKG nach EBM-Ziffer 03321 wird mit 20,54 EUR vergütet [5].

\section{Honorarverteilung Akupunktur auf einzelne Fachgruppen}

Der Honorarbericht der KBV für das 2. Quartal 2012 [9] führt nicht nur die Umsätze, sondern auch die 30 am häufigsten abgerechneten Gebührenpositionen der einzelnen Fachgruppen auf. Auf Grundlage dieser Daten lässt sich ein Einblick in die Verteilung von Akupunkturleistungen auf die einzelnen Fachbereiche gewinnen (Tab. 2).

Hieraus kann beispielsweise abgelesen werden, dass fast $46 \%$ aller Orthopäden Akupunkteure sind, dass pro 100 Patienten 22,7 Akupunkturbehandlungen erbracht werden und ein akupunktierender Orthopäde damit einen Umsatz von 12874 EUR erwirtschaftet, was fast $24 \%$ des durchschnittlichen Gesamtumsatzes entspricht. Demgegenüber wenden nur 11,38 \% der Allgemeinärzte Akupunktur an, in einer Häufigkeit von 1,8 pro 100 Patienten, und erwirtschaften mit 2091 EUR 4,39\% ihres durchschnittlichen Quartalsumsatzes.

Ein Blick auf die durch Akupunkturleistungen ausgelösten Gesamtumsätze der Fachgruppen zeigt, dass Orthopäden im Vergleich zu Allgemeinärzten einen um 227\% höheren Umsatz erzielen, obwohl die Anzahl der akupunktierenden Orthopäden nur 37\% der akupunktierenden Ärzte ausmacht. Akupunkturbehandlungen bei Patienten der GKV werden also vornehmlich in orthopädischen Praxen erbracht.

\section{Diskussion}

Drei Themenbereiche sollen beleuchtet werden: 1. Die Problematik des G-BA-Beschlusses zur Durchführung von Akupunkturbehandlungen und zu Qualifizierungsanforderungen; 2. Wirtschaftliche Aspekte von Akupunkturleistungen in der GKV; 3. Auswirkungen auf Akupunktur als Behandlungsmethode.

\section{Die Problematik des Beschlusses des Gemeinsamen Bundesaus-} schusses der Ärzte und Krankenkassen

Akupunkturbehandlungen in der GKV unterliegen Einschränkungen: 1. Verbot der Anwendung elektrischer Stimulationsakupunktur (ESA), 2. Beschränkung auf Akupunktur des Leitbahnsystems am Körper unter Ausschluss von Mikrosystemen und 3. Festlegung der Nadelanzahl pro Behandlung und Körperteil.

Diese Vorgaben sind dem Studiendesign der Modellprojekte geschuldet. Die Einbindung der ESA war von Beginn an vom G-BA ausdrücklich ausgeschlossen worden. In der Studienplanung der verschiedenen Modellprojekte wurden ausschließlich Körperpunkte definiert. Die Anzahl der Nadeln ist gemäß der Methodik der GERAC-Studien ermittelt, in denen für lumbale Schmerzen 14-20 Nadeln und für Schmerzen durch Gonarthrose 7-15 Nadeln festgelegt wurden [3].

Diese teilweise Standardisierung entspricht aber häufig nicht der Behandlungsrealität von akupunktierenden Ärzten. Vorhandene Therapiekonzepte werden individualisiert auch unter gleichzeitiger Verwendung von Mikrosystemen angewendet. Ohrakupunktur und Einführung in andere Mikrosysteme sind Lehrinhalte der «Zusatz-Weiterbildung Akupunktur» der Bundesärztekammer 
und auch Ausbildungsinhalt der Akupunktur-Fachgesellschaften [10-12]. Angaben zu Ober- und Untergrenzen der Nadelanzahl werden in dieser Form nicht gelehrt. ESA findet als zusätzliche schmerztherapeutische Option häufig Anwendung und ist auch Bestandteil der Akupunkturausbildung [11].

Die GKV-Akupunktur schränkt die Anwendung von Akupunktur ein und begrenzt durch Standardisierung weitere therapeutische Konzepte. Im Übrigen werden die Qualifikationsvoraussetzungen zur Erbringung von Akupunkturleistungen und die Anforderungen zur Qualitätssicherung von Akupunkteuren häufig als zu hoch empfunden. Um den umfangreichen Vorgaben zu entgehen, verzichten die Praktiker nicht selten auf die Kassenzulassung und erbringen Akupunktur ausschließlich privatärztlich.

Wirtschaftliche Aspekte von Akupunkturleistungen in der gesetzlichen Krankenversicherung

Die Vergütung von maximal 21,78 EUR für eine Akupunkturbehandlung ist unter Berücksichtigung der ärztlichen Behandlungszeit und der Belegung eines separaten Raumes über einen Zeitraum von mindestens 30 min nicht kostendeckend darstellbar. Innerhalb des EBM-Gefüges erscheint Akupunktur in Relation zu anderen Positionen wie Hausbesuchen dennoch nicht unterbewertet. Wie in vielen anderen Fachbereichen stehen auch ärztliche Akupunkteure unter erheblichem finanziellen und zeitlichen Druck.

Dass sich GKV-Akupunkturleistungen in orthopädischen Praxen häufen, muss nicht verwundern. Patienten mit chronischen Schmerzen der LWS und der Kniegelenke sind orthopädische Patienten. Auffallend ist jedoch, dass akupunktierende Orthopäden fast ein Viertel ihres Umsatzes durch Akupunktur generieren. Akupunkturleistungen stellen nach den Grundpauschalen (34,9\% des Umsatzes) mit großem Abstand zu allen anderen Leistungen die zweitwichtigste Umsatzquelle dar [9]. Dies dokumentiert eine große finanzielle Abhängigkeit orthopädischer Praxen von Umsatzerwartungen durch Akupunktur.

Bekannt ist, dass die Behandlungsergebnisse unabhängig von der Länge der Ausbildung des Akupunkteurs (A- oder B-Diplom) sind und dass der mittelfristige Erfolg am stärksten in denjenigen Praxen ausgeprägt ist, in denen neben Akupunktur auch andere Naturheilverfahren angeboten werden [13]
Interessant wäre zu untersuchen, inwieweit in der Routineversorgung Kostendruck, Zeitmangel, Umsatzerwartung und Fokussierung auf eine Behandlungsmethode die primär guten Ergebnisse der Akupunkturbehandlungen in den GERAC- und ART-Studien beeinflussen. Hierzu gibt es bisher keine Untersuchungen.

\section{Auswirkungen auf Akupunktur als Behandlungsmethode}

Patienten nehmen in Deutschland Akupunktur als Leistung der GKV in Anspruch. Sie haben es aber schwer, zwischen Akupunktur zu unterscheiden, die einer Standardisierung durch die GKV unterworfen ist und einer individualisierten Anwendung, die diesen Einschränkungen nicht unterliegt.

Damit besteht in der öffentlichen Wahrnehmung die Tendenz, Akupunktur weitgehend auf die vom G-BA angegebenen Indikationen zu reduzieren. Zwar fordern Patienten nicht selten Akupunkturbehandlungen zulasten der GKV bei anderen als den vorgesehenen Indikationen, weil sie das diffizile Verordnungswerk nicht kennen. Die breitere Anwendbarkeit auf andere Indikationen wie z.B. Migräne, Spannungskopfschmerz, allergische Symptome und vegetative Fehlregulationen tritt aber in den Hintergrund.

Zudem ist bei fehlender Wirksamkeit der GKV-Akupunktur die Bereitschaft groß, Akupunktur als Behandlungsmethode insgesamt für unwirksam zu erklären. Andere Akupunkturkonzepte als die der GKV-Akupunktur, die vielleicht besser geeignet gewesen wären, sind Patienten, die nach einem therapeutischen Fehlschlag enttäuscht wurden, nur schwer vermittelbar.

Aufseiten der Ärzteschaft führen zum einen die hohen Anforderungen zur Qualifizierung, zum anderen die Einschränkung in der Akupunkturbehandlung durch teilweise Standardisierung sowie der Kosten- und Zeitdruck zu einer bemerkenswerten Reaktion: Ärztliche Akupunkteure vermeiden bewusst die Beantragung, Akupunktur in der GKV erbringen zu können - selbst dann, wenn sie die Qualifizierungskriterien erfüllen.

\section{Disclosure Statement}

Der Autor erklärt hiermit, dass keine Interessenskonflikte in Bezug auf dieses Manuskript vorliegen.

\section{Literatur}

1 Zusammenfassender Bericht des Arbeitsausschusses «Ärztliche Behandlung» des Bundesausschusses der Ärzte und Krankenkassen über die Beratungen der Jahre 1999 und 2000 zur Bewertung der Akupunktur gemäß $\$ 135$ Abs.1 SGB V, 22.01.2001.www.g-ba.de/downloads/ 40-268-241/HTA-Akupunktur.pdf (Zugriff 23.02.2015).

2 NIH Consensus Conference: Acupuncture. JAMA 1998;280:1518-1524.

3 Zusammenfassender Bericht des Unterausschusses «Ärztliche Behandlung» des Gemeinsamen Bundesausschusses über die Bewertung gemäß $\$ 135$ Abs.1 SGB V der Körperakupunktur mit Nadeln ohne elektrische Stimulation bei chronischen Kopfschmerzen, chronischen LWS-Schmerzen und chronischen Schmerzen bei Osteoarthritis, 27.09.2007. www.g-ba.de/downloads/ 40-268-487/2007-09-27-Abschluss-Akupunktur.pdf (Zugriff 23.02.2015).
4 Bekanntmachung eines Beschlusses des Gemeinsamen Bundesausschusses über eine Änderung der Richtlinie Methoden vertragsärztliche Versorgung in Anlage I «Anerkannte Untersuchungs- oder Behandlungsmethoden» und in Anlage II «Methoden, die nicht als vertragsärztliche Leistungen zu Lasten der Krankenkassen erbracht werden dürfen»: Akupunktur. BAnz 2006;214:6952. www.g-ba.de/downloads/39-261-328/2006-09-19-RMvVAkupunktur-Massgabe_BAnz.pdf (Zugriff 23.02.2015).

5 Kassenärztliche Bundesvereinigung: Einheitlicher Bewertungsmaßstab (EBM), Stand 1. Quartal 2015. www. kbv.de/media/sp/EBM_Gesamt_Stand_1._Quartal_ 2015.pdf (Zugriff 23.02.2015).
6 Regelleistungsvolumina der KVH für das 3. Quartal 2013: QZV-Werte und Fallzahlen der Kassenärztlichen Vereinigung Hamburg. www.kvhh.net/media/public/ db/media/1/2012/06/501/qzv-werte-32013-1.korrekturlauf_stand27.01.2014.pdf (Zugriff 23.02.2015).

7 HzV-Vertrag gemäß \$73b Abs. 4 Satz 1 SGB V mit der TK in Bayern: Anlage 3 - Vergütung und Abrechnung. www.bhaev.de/images/stories/TK/Anlage_3_Vergtung und_Abrechnung_TK_Bayern.pdf (Zugriff 23.02.2015).

$8 \mathrm{HzV}$-Vertrag der TK gemäß \$73b Abs. 4 Satz 1 SGB V TK-Bayern: Anhang 1 zur Anlage 3, Stand 01. Juli 2014 HzV-Ziffernkranz. www.bhaev.de/images/hzv-vertraege/ Vertragsunterlagen/TK/2014_08_12_Anlage_3_Anhang_ 1_EBM_Ziffernkranz_HzV_TK_Bayern_gültig_ab_ 01_07_2014.pdf (Zugriff 23.02.2015). 
9 Kassenärztliche Bundesvereinigung: Honorarbericht für das zweite Quartal 2012. Zahlen und Fakten. www.

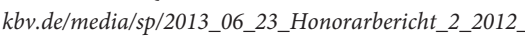
web.pdf (Zugriff 23.02.2015).

10 Bundesärztekammer: (Muster-)Kursbuch Akupunktur: Methodische Empfehlungen, Lehr- und Lerninhalte für den Grund- und Aufbaukurs der Zusatz-Weiterbildung «Akupunktur» gemäß der (Muster-)Weiterbildungsordnung 2003, Stand 25.06.2010, und den (Muster-) Richtlinien, Stand 18.02.2011, der Bundesärztekammer. www.bundesaerztekammer.de/downloads/MKBAkupunktur.pdf (Zugriff 23.02.2015).

11 Deutsche Ärztegesellschaft für Akupunktur (DÄGfA) Ärztliche Akupunktur 2015. www.daegfa.de/Documents/ Flipbook2015/ (Zugriff 23.02.2015).

12 Societas Medicinae Sinensis: Ausbildung Akupunktur, Akupunktur Kurs 7. www.tcm.edu/Aerztliche-Schule/ Ausbildung.de_Akupunktur.Akupunktur_Kurs_7.aspx (Zugriff 23.02.2015).

13 Weidenhammer W, Menz G, Streng A, et al.: Akupunktur bei chronischen Schmerzpatienten: Behandlungsergebnisse - Rolle des Akupunkteurs. Schmerz 2006;20:418:420-432. 